\title{
DEPORTE, TRADUCCIÓN Y SEMIOSIS
}

\author{
Pablo GARCÍA MATEO \\ (Universidad de Konstanz, Alemania)
}

Resumen: El presente artículo pretende describir, desde una perspectiva interdisciplinar, la interacción de aportaciones procedentes de la semiótica y traducción en las ciencias de la actividad física y el deporte. Tomando el referente de la traducción como responsable del trasvase de códigos comunicativos, se ve cuál es su importancia en las diferentes manifestaciones físico-deportivas que nos rodean. El objetivo, por tanto, es el de definir campos de interconexión entre estos fenómenos de distinta naturaleza. Partimos de la hipótesis de la existencia de un campo inexplorado de convergencia, que tratamos de comenzar a definir, de la forma más humilde posible.

Para ello proponemos tres niveles claramente diferenciados de conexión. La primera y más inmediata se registra dentro de la denominada traducción deportiva, que no es más que la traducción especializada en el ámbito deportivo, tal y como ya existe en otros ámbitos. En segundo lugar, definimos la traducción deportiva institucionalizada. Mediante esta se establecen formalmente equivalencias en la terminología deportiva, que suponen la regulación y homologación de una realidad deportiva. Dicha realidad se transcribe a la sociedad, con inmediatas consecuencias para la presencia efectiva de una disciplina deportiva en la vida social. Finalmente se analiza el papel de la traducción en la semiótica deportiva, estableciendo cinco niveles básicos de intervención: traducción semiótica formalizadora, decodificadora, reguladora, 


\title{
P. García Mateo \\ 2019. "Traducción, deporte y semiosis" \\ Sabir. International Bulletin of Applied Linguistics, 1: 110-132
}

resultativa y divulgativa. Todas ellas resultan esenciales en el desarrollo satisfactorio de la práctica deportiva.

Tras la presente investigación se refuerza la hipótesis en la que se sustenta este trabajo, concluyendo que existe un campo de convergencia interdisciplinar entre la semiótica y las ciencias de la actividad física y el deporte. La traducción forma el eje para explicar dicha intervención de la comunicación en el deporte. Este es un campo prácticamente inexplorado hasta la fecha, todavía por definir, pero de especial interés dada la relevancia de la comunicación en desarrollo satisfactorio de la práctica deportiva.

Palabras clave: Traducción, semiótica, deporte.

\begin{abstract}
This article aims to describe, from an interdisciplinary perspective, the interaction of semiotics' and translation's contributions in sport sciences. Taking the reference of translation as the responsible for the transfer of communicative codes, we will see its importance in the different physical and sportive manifestations that surround us. The aim, therefore, is to define fields of interconnection between these phenomena of different nature. We hypothesize the existence of an unexplored field of convergence, which we try to define in the most-humble possible manner.
\end{abstract}

For this, we propose three clearly differentiated levels of connection. The first and most immediate is known as sports translation. It is no more than the specialized translation in the sports field, as it already exists in other fields. Secondly, we define the institutionalized sports translation. This establishes formal equivalences in sports terminology, regulating and homologating the sporting reality. This reality is transcribed to society, with immediate consequences for the effective presence of a sporting discipline in social life. 


\section{P. García Mateo \\ 2019. "Traducción, deporte y semiosis" \\ Sabir. International Bulletin of Applied Linguistics, 1: 110-132}

Finally, the last of the sections analyses the role of translation in sports semiotics, establishing five basic levels of intervention: formalizing, decoding, regulating, resultant and informative semiotic translation. All of them are essential in the satisfactory development of the sports activity.

The present research reinforces the hypothesis on which this work is based. It is concluded that there is an interdisciplinary field of convergence between semiotics and sport sciences, taking translation as an axis to explain this intervention of communication in sport. This is, without a doubt, a field practically unexplored to date, yet to be defined. Nevertheless, it has special interest given the relevance of the communicative processes for the satisfactory development of sports practice.

Keywords: Translation, semiotics, sport.

\section{Previo}

El presente artículo forma parte de una investigación más amplia que desarrollo junto con el área de Lingüística General de la Universidad de Almería, donde cursé mis estudios de Ciencias de la Actividad Física y el Deporte. En ella se examinan las conexiones que vinculan comunicación y deporte, dentro de una perspectiva interdisciplinar que combina aportaciones procedentes de la lingüística aplicada, la semiótica y la propia ciencia del deporte. En abril de 2018 se planteó la conveniencia de incorporar un nuevo eje de observación procedente de la traducción. La propuesta, sin duda sugerente, surgió del continuo diálogo científico que, desde hacía décadas, mantenían los lingüistas de Almería y los traductores de Málaga. La traducción, en tanto que responsable del trasvase de códigos comunicativos, por fuerza había de estar implicada, en el grado y medida que fuese, dentro de la actividad física. Ese esbozo de idea contó con el inmediato 


\section{P. García Mateo \\ 2019. "Traducción, deporte y semiosis" \\ Sabir. International Bulletin of Applied Linguistics, 1: 110-132}

respaldo y el firme entusiasmo de Emilio Ortega Arjonilla. No vaciló un instante en animar con entusiasmo una perspectiva que, con independencia del resultado final al que llegase, merecía cuando menos ser explorada. Meses después recibíamos desde Málaga la tristísima noticia de su repentino fallecimiento. Este trabajo, pues, heredero evidente de muchos de sus planteamientos interdisciplinares, pretende sumarse a su homenaje, por razones científicas, pero también personales. Tuve la fortuna de conocer antes al Emilio persona, a una humanidad afable y afectuosa. Después he descubierto al Prof. Ortega Arjonilla, a pesar de nuestra distancia disciplinar. Ambos me han parecido extraordinarios.

\section{La traducción de las lenguas del deporte}

La primera y más inmediata conexión se registra, como por otra parte cabía esperar, dentro del dominio que podría denominarse traducción deportiva. Existiría una traducción propia de ese ámbito, de la misma forma que lo hacen la traducción médica, jurídica, económica, literaria, etc. Formaría parte, en consecuencia, de lo que se conoce como traducción especializada que, para empezar, debe partir de un solvente trabajo terminológico (Cabré, 2004).

Las previsiones de Cabré se cumplen estrictamente en el dominio deportivo. Se ha consignado una continuada preocupación por aquilatar con exactitud la terminología deportiva, sobre todo en lo tocante a los instrumentos de medición empleados por los especialistas. De la corrección, o no, de las traducciones empleadas para adaptar las escalas de una lengua a otra dependía, en última instancia, el éxito de estas, su correcta aplicación en la práctica. Esa preocupación y esa inquietud no han conocido prácticamente restricciones de lengua $\mathrm{o}$ de contextos culturales. Se ha querido verificar los protocolos 


\section{P. García Mateo \\ 2019. "Traducción, deporte y semiosis" \\ Sabir. International Bulletin of Applied Linguistics, 1: 110-132}

empleados por esas traducciones en español (Balaguer et al., 1996; Walle, 2002; Araya et al., 2009), en francés (Ferreira, 2017), en inglés (Bohu et al., 2015), en turco (Harput et al., 2017), en italiano (Sartorio et al., 2014) o en alemán (Elbe, 2004; Elbe et al., 2005; Stiler y Alferman, 2007; Zeech, 2013). En ocasiones se ha medido con mayor detalle la dislocación cultural que ello suponía (Ruiz y Watt, 2012; Hamadou, 2010; Stenlling, 2014), subrayando que no se trata solo de una cuestión de transferencia terminológica, sino también de traslación de categorías conceptuales y cognoscitivas. Como se puede comprobar, se trata de una línea de preocupaciones relativamente recientes, aunque progresivamente creciente y cada vez más asentada en la bibliografía.

Otras preocupaciones vinculadas a la traducción y al deporte han tenido una aparición considerablemente más ocasional, en término generales, han carecido de una continuidad semejante a la del caso anterior. Montero (2009) trazó las posibles equivalencias de las metáforas futbolísticas en español e italiano. En otras ocasiones se han revisado traducciones concretas de libros de referencia dentro del ámbito de la actividad física y el deporte. Procházková (2006) revisó la versión francesa de L'Histoire du sport en Europe que L'Harmattan había publicado dos años antes. Loudcher (2008) repitió la experiencia, en esta ocasión a propósito de la traducción del From Ritual to Record: the Nature of Modern Sports de Guttman, también al francés. Algunas historias del deporte (Fortuin, 1996; Salvador, 2009) del deporte no han olvidado incluir la evolución de sus nomenclaturas a lo largo del tiempo. La literatura también ha propiciado interpretaciones análogas, siempre subrayando las diferencias conceptuales que implican los contextos de los que se forma parte (Sonnenschein, 1980; Febles, 2000).

Más ocasionalmente se han abordado problemáticas particulares de algunos deportes, caso del hockey hielo (Mrazik 2011; Richmond et al., 2013), los 


\section{P. García Mateo \\ 2019. "Traducción, deporte y semiosis" \\ Sabir. International Bulletin of Applied Linguistics, 1: 110-132}

deportes formativos (Holt et al., 2018) o las dinámicas entre las que se desenvuelven los entrenadores (Pope, 2015). Este último ámbito lo vinculó García Marcos (2018) a la lingüística aplicada con fines específicos, solo que, trasladado a los dominios de la interpretación, más que a los de la traducción. El matiz de García Marcos no dejaba de introducir una puntualización técnica. La oralidad, en efecto, parece contener mayor número de interacciones en las que se registran esa clase de intercambios lingüísticos entre deportistas y entrenadores. Pero, indirectamente, abría un campo de expectativas nuevas para la temática que aquí se toca.

Por último, Keller (2018) propone una reflexión muy actual, también muy indicativa de la situación social en la que se encuentra el deporte actual. Keller plantea si existe la posibilidad de considerar la traducción deportiva como un campo de plena especialización. La razón que inclina finalmente la balanza en la dirección positiva es tan determinante como que constituye una especialización económicamente rentable, con un mercado capaz de surtir de suficiente demanda a los profesionales. En definitiva, la enorme repercusión social que ha adquirido en las últimas décadas no ha hecho más que proyectarse sobre un dominio más de intervención social, la traducción en este caso.

\section{La institucionalización de la traducción deportiva}

El deporte tiene otro frente abierto en relación con la traducción. Mediante el Decreto 163/2010, de 22 de junio, el Gobierno de España regula formalmente los criterios por los cuales han de regirse los nombres de los clubes deportivos. No es una cuestión superficial, ni mucho menos; tampoco es asunto exclusivo de España. Es preciso ajustar la terminología deportiva por múltiples razones. Para 


\section{P. García Mateo \\ 2019. "Traducción, deporte y semiosis" \\ Sabir. International Bulletin of Applied Linguistics, 1: 110-132}

empezar, resulta determinante para adscribir los deportes a las federaciones, adscribirlos a los correspondientes comités olímpicos y, a resultas de ello, organizar y formalizar sus competiciones. Quizá en los deportes mayoritarios esta pueda resultar una cuestión relativamente secundaria, aunque solo "relativamente". No hay dudas mayores acera de qué es el "fútbol". Pero sí es determinante determinar qué conexiones tiene la modalidad disputada en pabellones, si es "futbito", si es "fútbol-sala" o "futsal", si es similar al "fútbol 7", o si pertenece a un ámbito distinto. En función de cuál sea la resolución de esos interrogantes, esa modalidad deportiva se incluirá -o no- dentro de las correspondientes federaciones de fútbol, podrá optar a convertirse en deporte olímpico, recibirá unos u otros patrocinios $y$, en definitiva, estará en condiciones de asumir una u otra proyección social.

Si eso es así en el dominio de una lengua, su traslación al ámbito internacional resulta más acuciante. Alías García (2014) planteaba esa duda en relación con los deportes náuticos, en el marco de una investigación de más amplio alcance sobre la percepción de los mismos en la comunidad de Almería. Tres años después (Alías, 2017), presentaba un vocabulario de terminología de los deportes náuticos que pretendía homologar la versión española con los estándares en la bibliografía y, en sentido amplio, con las instituciones deportivas a nivel internacional.

Alías detectaba una urgencia que no era exclusiva de los deportes náuticos, ni tan siquiera del deporte en sentido amplio. Los deportes mayoritarios disponían de una amplia equivalencia terminológica entre lenguas, fundamentalmente avalada por el uso, por la tradición de años siendo empleado masivamente. El "deuce" tenístico en inglés se había traducido como "iguales" en español o como "egalité" en francés. A veces incluso ni se había traducido, incorporando directamente el extranjerismo. Mientras que el español traduce el 


\section{P. García Mateo \\ 2019. "Traducción, deporte y semiosis" \\ Sabir. International Bulletin of Applied Linguistics, 1: 110-132}

"offside" inglés como" fuera de juego" (no siempre, por cierto), en alemán ha aceptado la variante inglesa, incorporándola directamente. La situación varía significativa y drásticamente en lo tocante a los deportes minoritarios, como señalaba Alías.

Indefinición terminológica implica la imprecisión en la adscripción a una u otra federación y, por consiguiente, lastra la proyección de la actividad deportiva en cuestión. ¿El "bodyboarding" está dentro o fuera del "windsurfing"? Y a renglón seguido, ¿dentro de qué federación se incluye? En España forma parte de la Federación Española de Surf, pero en Chile sin embargo tiene una federación propia. Existen un circuito y un campeonato mundiales de bodyboarding autónomos, pero al mismo tiempo parece que ello implica menor proyección olímpica, con lo que supone en última instancia quedar fuera de esos circuitos deportivos y comerciales.

La propuesta de Alías (2017) está encaminada en esa dirección, en la de tratar de paliar esas distancias, ofreciendo alternativas de traducción fiables que permitan establecer equivalencias susceptibles de ser homologadas $y$, en consecuencia, que ofrezcan tablas de equivalencia oficiales.

La inquietud de Alías, en todo caso, no era nueva ni estaba desconectada de iniciativas institucionales de más hondo calado y recorrido. El Comité Olímpico Internacional dispone de su propio catálogo de equivalencias en terminología deportiva, preocupación que han compartido otras instituciones encargadas de velar por la planificación de las principales lenguas del planeta, caso de la Fundeu para el español.

Establecer formalmente las equivalencias en la terminología deportiva, sin duda, supone una homologación que va más allá de la terminología. En última instancia regula una realidad que es la transcrita a la sociedad, con las consecuencias derivadas de ello en forma de organización de eventos, 


\section{P. García Mateo \\ 2019. "Traducción, deporte y semiosis" \\ Sabir. International Bulletin of Applied Linguistics, 1: 110-132}

negociación de patrocinios, cuotas de programación y, en definitiva, presencia efectiva en la vida social.

\section{La traducción como hermeneusis semiótica en el deporte}

Resta un último apartado, probablemente el más sugerente, aunque es necesario adentrarse en él con prudencia y plantearlo en términos estrictamente hipotéticos. La bibliografía especializada sobre traducción subraya que esta no se limita única y exclusivamente al trasvase de contenidos de una lengua a otra. En primer lugar, porque en realidad se reinterpreta lo manifestado en la lengua traducida. Pero, en segundo, porque la operación de traducción puede concebirse en términos más amplios. Tanto es así que cabe la posibilidad de concebir un diccionario como un gran mecanismo de traducción de una lengua hacia sí misma: una palabra (la entrada) es reinterpretada por otras que la explican ${ }^{1}$. Ortega Arjonilla (1995) había profundizado un grado más en su tesis doctoral, un trabajo histórico que más de un cuarto de siglo después de su defensa sigue aportando sugerencias, incluso para disciplinas como la ciencia de la actividad física y el deporte, en apariencia tan alejadas. Ortega Arjonilla realiza ahí un trabajo teórico y metodológico de neta proyección interdisciplinar. Parte de tres ámbitos de conocimiento: la hermenéutica, la traductología y la teoría de la comunicación, desde los que construye una herramienta de aplicación con carácter general para cualquier proceso de reinterpretación y traslación de significados. Tanto es así que ejemplifica su propuesta analizando cómo se traduce "El nombre de la rosa" de lenguaje literario a lenguaje fílmico.

\footnotetext{
${ }^{1}$ Quiero agradecer muy sinceramente a N. Perdu la explicación de este concepto, no tan fácil ni inmediato para quien, como yo, procede de una disciplina lejana y, en definitiva, está iniciando su andadura en el ámbito de la investigación.
} 


\section{P. García Mateo \\ 2019. "Traducción, deporte y semiosis" \\ Sabir. International Bulletin of Applied Linguistics, 1: 110-132}

En mi modesta opinión, esa propuesta de Ortega Arjonilla está dotada de una enorme potencialidad, para explicar multitud de procesos de traducción conforme a esa acepción amplia que formula. El deporte no es una excepción, o cuando menos esa es una hipótesis que desearía discutir aquí. De partida sería posible plantear cinco niveles de traducción semiótica dentro de la práctica deportiva.

La traducción semiótica formalizadora se encarga de plasmar ideas y planteamientos abstractos en formatos que puedan ser entendidos y aplicados por el colectivo al que se dirigen. Esa es la función que ejercen sobre todo los entrenadores, cuando instruyen a sus equipos o pupilos en las tácticas y estrategias que han de emplearse. Para ello es habitual que acudan al canal verbal dando instrucciones, corrigiendo posiciones, recordando consignas o insistiendo en planteamientos ya comentados. El lenguaje verbal no es el único formato posible de destino de este tipo de traducción deportiva. Las conocidas pizarras de los entrenadores, en prácticamente todos los deportes colectivos, trasladan la estrategia de los entrenadores a movimientos concretos del equipo. La exacta comprensión de las mismas resulta determinante para el desempeño del juego en general, cuya pauta determinan. De la correcta colocación y el adecuado desplazamiento de los jugadores depende buena parte del resultado que obtengan al final de sus partidos.

La semiótica deportiva decodificadora es el complemento imprescindible del tipo anterior. Mediante ella, el deportista traduce a movimientos las instrucciones de sus entrenadores o lo plasmado en la pizarra. Una flecha que recorre todo un campo de fútbol en paralelo a una línea de lateral ha de convertirse en un "carrillero" tratando de avanzar por la banda durante un partido. De nuevo los deportes colectivos suministran la mayoría de la casuística en este sentido, con ejemplos análogos al comentado que pueden proceder del 


\section{P. García Mateo \\ 2019. "Traducción, deporte y semiosis" \\ Sabir. International Bulletin of Applied Linguistics, 1: 110-132}

balonmano, el baloncesto, el voleibol o, entre muchos otros, el waterpolo o el rugby.

Como se ha señalado, estos dos tipos de traducción semiótica deportiva actúan de manera conexa, estableciendo una secuencia que puede retroalimentarse e iniciar de nuevo el circuito. Un entrenador traduce un planteamiento táctico en forma de dibujo sobre una pizarra. Sus pupilos trasladan esa información durante el desarrollo del juego. Pero si se produce algún error de interpretación, si no se actúa conforme a lo previsto y diseñado previamente, el entrenador vuelve a iniciar un nuevo ciclo de formalización, recuerda instrucciones y corrige el desarrollo de lo que está sucediendo sobre el terreno de juego.

Esa secuencia se hace singularmente perceptible en las llamadas jugadas de estrategia, un episodio deportivo intensamente vinculado a procesos muy exactos de traducción semiótica. EL éxito de las mismas radica, al menos en gran medida, en que los preparadores sean capaces de transmitir la secuencia de movimientos que debe desarrollar su equipo en una jugada puntual. Las faltas y los saques de esquina en fútbol, las "touche" en rugby, los golpes francos en balonmano o los saques de fondo del baloncesto suministran ejemplos prototípicos al respecto. En todos ellos, la posibilidad de concluir con éxito conforme a lo previsto por los técnicos es directamente proporcional a la exactitud interpretativa que sean capaces de desarrollar los jugadores.

Existe una segunda diada, en esta ocasión prioritariamente derivada del desarrollo del juego. Mientras que los dos tipos anteriores son, en gran medida, fruto de la planificación y la programación del deporte, estas dos nuevas modalidades surgen, o no, al ritmo que va marcando la propia actividad. De una parte, aparece lo que se denominaría traducción semiótica deportiva reguladora, asignada de modo prácticamente exclusivo a los jueces de los acontecimientos 


\section{P. García Mateo \\ 2019. "Traducción, deporte y semiosis" \\ Sabir. International Bulletin of Applied Linguistics, 1: 110-132}

deportivos. Las infracciones que se cometen son traducidas en forma de sanción, para cuya comunicación existen códigos especializados. Estos, a su vez, informan a los contendientes mediante un nuevo proceso de traducción, que requiere de elementos simbólicos compartidos y conocidos por los jueces y los deportistas. Es más, ese conocimiento es requisito para la práctica deportiva correspondiente. El balonmanista $A$ empuja en el aire al balonmanista $B$, que en ese momento estaba tratando de realizar un lanzamiento. Los árbitros traducen esa infracción haciendo sonar el silbato. Señalan de inmediato el punto de lanzamiento de siete metros hacia el que se desplazan. A continuación, levantan el brazo con únicamente los dedos índice y corazón extendidos, situándose frente al balonmanista $A$, lo que indica que es excluido durante dos minutos. Los colegiados de rugby indican los golpes de castigo, marcando con el brazo extendido hacia la zona del equipo beneficiario del mismo. Al mismo tiempo, traducen la infracción cometida mediante código gestual y, por lo general, explicaciones verbales. Por el carácter regulador de estas traducciones semióticas en el deporte es preciso que hayan sido establecidas de manera formal, regulada y explícita. Lo más normal es que figuren en las correspondientes reglamentaciones de cada especialidad.

Con todo, hay posibles usos reguladores de la traducción semiótica deportiva no sujetos exactamente al arbitraje, o en todo caso fuera del campo de las infracciones propiamente dichas. La bandera amarilla en Fórmula 1 informa de un peligro para el desarrollo de la carrera que va a obligar a neutralizarla. Los banderines en los estadios de fútbol, las líneas de fondo o de lateral en un pabellón o en otros terrenos de juego o, entre otros, las líneas que marcan las calles en una carrera de cien metros lisos traducen los límites entre los que discurre la competición. Para cumplir esos cometidos se acude a una diversidad ostensible de recursos de diferente naturaleza semiótica: físicos (líneas de marca), cromáticos (colores de los banderines), proxémicos (habilitando y prohibiendo la 


\section{P. García Mateo \\ 2019. "Traducción, deporte y semiosis" \\ Sabir. International Bulletin of Applied Linguistics, 1: 110-132}

utilización de espacios). En ocasiones, se produce una interacción simultánea en varios niveles de semiosis. Hay acciones que traducen un momento de la competición, lo dejan patente, pero además contienen también fuerza ilocutiva; esto es, forman parte de la dinámica deportiva en sí misma. El pitido final con el que concluye un partido traduce la realidad que muestran los marcadores de los estadios o pabellones, informan a los participantes y a los espectadores de esa circunstancia, pero a la vez son el momento exacto de la finalización del encuentro. Técnicamente este no concluye hasta que no se produce ese pitido final.

La consecuencia y el complemento del último tipo surgiría con la traducción semiótica deportiva resultativa que vierte las prescripciones indicadas mediante la anterior en las correspondientes actuaciones que se derivan de ellas. Tras el pitido final al que acaba de aludirse, los deportistas traducen sus consecuencias y se encaminan al túnel de vestuarios. Los dos dedos del árbitro de balonmano al aire equivalen a exclusión y son transcritos como retirada al banquillo por parte del jugador señalado. Tres avisos a un marchador atlético han de ser equivalentes a su exclusión. La casuística, de nuevo, es extensa, o en realidad prácticamente inagotable. Esta clase de traducción semiótica deportiva requiere de la existencia previa de la anterior, respecto de la que es una consecuencia directa y necesaria, reglada de manera precisa y exhaustiva. Un jugador de fútbol solo abandona un terreno de fútbol sí, y solo sí, el colegiado del encuentro que disputa le muestra una tarjeta roja. El que lo hiciera un entrenador u otro rival, por mencionar ejemplos extremos, carecería de efecto alguno $y$, en último término, sería interpretado en términos sustancialmente distintos (como una provocación, una humorada, etc.) Tampoco carecería de obligatoriedad si quien la mostrase fuese un "linier", lo que se traduciría como una impropiedad en la aplicación del reglamento. Se trata de comunicaciones muy regladas, como se ha comentado hace un instante, con una traducción 


\section{P. García Mateo \\ 2019. "Traducción, deporte y semiosis" \\ Sabir. International Bulletin of Applied Linguistics, 1: 110-132}

unívoca y obligatoria. La tarjeta roja en fútbol solo puede interpretarse como expulsión y el jugador no puede negarse a ella, obviar su contenido. Después de recibirla ha de abandonar siempre y sin excepción el juego.

Existe un último tipo, la traducción semiótica deportiva divulgativa, cada vez más empleada y habitual en los medios de comunicación. En esta ocasión se traslada a grafismo visual lo que ha sucedido, antes, durante o después de una retransmisión deportiva. Los movimientos tácticos de los equipos, sus diferentes disposiciones estratégicas, los movimientos desarrollados en momentos puntuales o jugadas especiales terminan sintetizados en una pizarra digital que es comentada a los espectadores. En ocasiones se marcan esas mismas líneas de la disposición de un equipo sobre la propia imagen emitida en tiempo real. Este es un recurso cada vez más habitual en las retransmisiones de partidos de fútbol, sobre todo para subrayar los dibujos tácticos entre los que están desempeñándose los equipos.

Se emplean, además, otros sistemas de traducción a otros lenguajes visuales y no visuales- que están cada vez más extendidos, hasta el punto de que en algunos deportes se han convertido en una de sus señas de identidad. Las estadísticas traducen a notación numérica lo que ha sucedido en el transcurso de un encuentro deportivo. Aportan una extraordinaria capacidad para condensar la información de lo sucedido, traduciendo de manera sincrética y objetiva (gracias a los números) un encuentro. No parece, en todo caso, que sea una traducción completa y exhaustiva. Las estadísticas reflejan solo la parte de la realidad deportiva, la susceptible de ser cuantificada. Fuera quedan los elementos cualitativos que pueden resultar determinantes en el desarrollo de un acontecimiento deportivo. Por lo demás, la cuantificación no discrimina tipos contextos y situaciones que sí son relevantes en la práctica deportiva. No es lo mismo que el futbolista A promedie un $90 \%$ de pases acertados a dos metros, 


\section{P. García Mateo \\ 2019. "Traducción, deporte y semiosis" \\ Sabir. International Bulletin of Applied Linguistics, 1: 110-132}

que el futbolista B mantenga esa cifra, pero enviando el esférico a 30 o 40 metros de distancia.

No obstante, como se han avanzado, algunos deportes han incorporado de manera decisiva y firme las estadísticas, con varios niveles de uso, además. En baloncesto constituyen una referencia obligada, con índice propios que traducen el rendimiento global de un jugador. La conocida valoración que cada uno de ellos obtiene al final de un partido, pondera todos los aspectos del juego, con lo que aporta un índice muy relevante del rendimiento de cada baloncestista. Son referencias extraordinariamente apreciadas de las que depende en gran medida la imagen que transmite cada jugador ante los medios de comunicación y ante el gran público en general. Pero esos mismos índices pueden ser empleados como información interna por los preparadores y los equipos para dar cuenta del rendimiento de sus pupilos, valorar sus actuaciones y corregir sus errores. Sucede así en el baloncesto, pero también en deportes que han desarrollado medidas análogas, caso del balonmano.

Hay, por lo demás, aspectos del deporte que no pueden recoger las estadísticas. Sin salirse del baloncesto, algunos componentes determinantes del desarrollo del juego no son susceptibles de ser traducidos numéricamente. La ductilidad para llegar a las ayudas, la rapidez en el pase, la empatía con el grupo, la capacidad para bloquear el rebote o la precisión en los ajustes de una zona difícilmente encuentran una formulación numérica $y$, por tanto, no admiten transcripción estadística.

En otras ocasiones la traducción se realiza a lenguajes meramente gráficos. Los medios de comunicación también acuden con mayor frecuencia a los gráficos de presencia de un deportista sobre un terreno de juego, en los que se colorea con diversa intensidad los espacios entre los que se ha desenvuelto durante un 


\section{P. García Mateo \\ 2019. "Traducción, deporte y semiosis" \\ Sabir. International Bulletin of Applied Linguistics, 1: 110-132}

partido. Tienen un gran rendimiento, tanto en la televisión, como en la prensa deportiva gráfica.

La traducción semiótica deportiva divulgativa no deja de tener un componente reiterativo, aclaratorio o sintetizador respecto a la información que han recibido los participantes en un evento deportivo, sus preparadores o el público que lo sigue. En realidad, condensa información sobre acontecimientos que ya deben haber sido visto y vividos. Si durante un partido de baloncesto un alero se ha mostrado infalible, las estadísticas le concederán un porcentaje de tiro espectacular, que en el fondo no hará más que confirmar la vivencia que ha tenido el espectador de que ha resultado decisivo durante el transcurso del partido. Las estadísticas pueden corroborarlo, pero en todo caso cualquier aficionado al rugby sabe, porque lo ha visto, que Beauden Barret es, probablemente, el jugador más determinante del panorama actual. En ese sentido, la traducción semiótica deportiva divulgativa incorpora un elemento muy apreciado por la actual comunicación de masas. Retoma en diferido la comunicación ya conocida para someterla a análisis más minuciosos, más detenidos, también más polémicos habitualmente. Tanto es así que los formatos de la programación deportiva, sobre todo en los medios audiovisuales, aunque en parte también en los escritos, se ha acomodado a estos nuevos recursos, incluso como uno de sus grandes reclamos de atención para el gran público. De ese modo, han creado productivos comunicativos propios, especializados en el análisis de los acontecimientos deportivos, para lo que emplean estos recursos. Esa programación consigue diferenciarse de las retransmisiones, precisamente gracias a las posibilidades de recuperación y análisis de la información que facilita la traducción semiótica deportiva divulgativa. 


\section{Conclusiones}

El deporte está surcado de innumerables coyunturas en las que se generan procesos que requieren recodificar y descodificar mensajes, verterlos de un lenguaje a otro, en definitiva, traducirlos semánticamente. La hipótesis de partida de esta investigación, pues, se ha confirmado en términos generales y, con toda la prudencia necesaria, pondera la posibilidad de continuar por esa dirección en el futuro.

No obstante, también es evidente que concita fenómenos de distinta naturaleza, no necesariamente interconectados entre sí. La traducción y la terminología deportivas introducen dentro de dominios vinculados a la traductología y a la lingüística aplicada. Los tipos de traducción semiótica deportiva, por su parte, están mucho más vinculados a esa dimensión heurística entre códigos que había planteado Ortega Arjonilla. De hecho, son un refrendo empírico, procedente de un mundo casi impensable, a su hipótesis nuclear.

En esos tres niveles, o grados si se prefiere, de trabajo sí que existe un gran denominador común como es la presencia necesaria de especialistas en ciencias de la actividad física y el deporte. Por lo tanto, se plantea un horizonte inequívocamente interdisciplinar, con convergencia de especialista en esta misma disciplina, además de lingüistas, semióticos y traductores, como mínimo.

Por otra parte, esta aproximación a la traducción semiótica dentro del ámbito deportivo refuerza la hipótesis marco en la que se sustenta este trabajo. En efecto, parece existir un amplio -e inexplorado- campo de convergencia interdisciplinar entre la semiótica y las ciencias de la actividad física y el deporte. A través de él, debería ser posible dar cuenta de la intervención de la 


\section{P. García Mateo \\ 2019. "Traducción, deporte y semiosis" \\ Sabir. International Bulletin of Applied Linguistics, 1: 110-132}

comunicación en el deporte. La traducción, en cualquiera de las acepciones aquí examinadas, supone uno de los posibles ejes de pertinencia en esa dirección. Pero, en todo caso, no deja de ser un aspecto más dentro de un elenco ostensiblemente más amplio. Las investigaciones deportivas sobre kinésica, proxémica, cromémica o imaginario social deberían encontrar el camino expedito, siempre dentro de dinámicas interdisciplinares de investigación, acogidas prioritariamente a paradigmas dinámicos $\mathrm{y}$, en la medida de lo posible, tendencialmente caotológicos. Ninguna realidad parece ya discreta. La que se atreve a examinar la convergencia entre los sistemas semióticos de comunicación y el deporte, como parece obvio, tampoco lo es.

\section{Bibliografía}

Alías, A.

2014. Comunicación y deporte. los deportes náuticos entre los estudiantes de la UAL. Almería: Universidad de Almería.

2017. Vocabulario enciclopédico de deportes náuticos. Almería: Parra ed.

Araya-Vargas, G. A.; Gapper-Morrow, S.; Moncada-Jiménez, J. y J. Buckworth.

2009. "Translation and cross-cultural validation of the Spanish version of the Mindful Awareness Attention Scale (MAAS): An exploratory analysis and potential applications to exercise psychology, sport and health" en International Journal of Applied Sports Sciences, 21(1): 94-114.

Balaguer, I., Castillo, I. e I. Tomás.

1996. "Análisis de las propiedades psicométricas del Cuestionario de Orientación al Ego y a la Tarea en el Deporte (TEOSQ)" en Psicológica, 17: 71-81.

Bohu, Y., Klouche, S., Lefevre, N., Webster, K. y S. Herman, S. 
2015. "Translation, cross-cultural adaptation and validation of the French version of the Anterior Cruciate Ligament-Return to Sport after Injury (ACL-RSI) scale" en Knee Surgery, Sports Traumatology, Arthroscopy, 23(4): 11921196.

Cabré, M. T.

2004. "La terminología en la traducción especializada". Gonzalo, C. u García Yebra, V. (eds.) Valentín. Manual de documentación y terminología para la traducción especializada. Madrid: Arco Libros, 89-125.

Elbe, A. M.

2004. "Testgütekriterien der deutschen Version des Sport Orientation Questionnaires" en Spectrum, 16(1): 96-107.

Elbe, A. M.; Wenhold, F. y D. Müller.

2005. "Zur Reliabilität und Validität der Achievement Motives Scale-Sport" em Zeitschrift für Sportpsychologie, 12(2): 57-68.

Durand, M., Cury, F., Sarrazin, P. y J. P. Famose.

1996. "Le Questionnaire de Perception du Succès en Sport: Validation française du" Perception of Success Questionnaire" en International Journal of Sport Psychology, 27 (3): 251-268.

Febles, J.

2000. "Martí frente a dos deportes anglosajones: Antagonismo conceptual y traducción hermética en algunas Escenas norteamericanas" en Hispania, 83 (19): 19-30.

Ferreira, I. 
2017. "Sport à tout prix? Dépendance à l'exercice physique et soins de santé primaires en contexte français: traduction française et validation de I'Exercise Addiction Inventory." Grenoble: Univ. Grenoble. Consulta: https://dumas.ccsd.cnrs.fr/dumas-01528930/document.

Fortuin, R. W.

1996. Der Sport im augusteischen Rom: philologische und sporthistorische Untersuchungen (mit einer Sammlung, Übersetzung und Kommentierung der antiken Zeugnisse zum Sport in Rom) . Zurich: Franz Steiner Verlag.

García Marcos, F.

2018. La trastienda de la enseñanza de lenguas extranjeras. Granada: Comares.

Hamadou, A. B.; Piton, O. y H. Fehri.

2010. Recognition and translation Arabic-French of Named Entities: case of the Sport places. Consulta https://arxiv.org/ftp/arxiv/papers/1002/1002.0481.pdf.

Harput, G.; Tok, D.; Ulusoy, B.; Eraslan, L.; Yildiz, T. I.; Turgut, E. y N. Ergun.

2017. "Translation and cross-cultural adaptation of the anterior cruciate ligamentreturn to sport after injury (ACL-RSI) scale into Turkish" en Knee Surgery, Sports Traumatology, Arthroscopy, 25(1): 159-164.

Holt, N. L.; Camiré, M.; Tamminen, K.; Pankow, K.; Pynn, S. R.; Strachan, L. y J. Fraser-Thomas.

2018. "PYDSportNET: A knowledge translation project bridging gaps between research and practice in youth sport" en Journal of Sport Psychology in Action, 9(2): 132-146.

Keller, J. 
2018. "Tu deviens traducteur sportif parce que t'as des clients qui travaillent dans le domaine du sport»: la traduction sportive, un type de traduction spécialisée?'. Ginebra: Univ. Généve. Tesis Doctoral.

Loudcher, J. F.

2008. "À propos de la traduction française du livre de Allen Guttmann, From Ritual to Record: the Nature of Modern Sports" en Staps, 2: 39-51.

Mrazik, M.; Bawani, F. y A. L. Krol.

2011. "Sport-related concussions: knowledge translation among minor hockey coaches" en Clinical Journal of Sport Medicine, 21 (4): 315-319.

Montero, J. F. M.

2009. "La metáfora en el léxico futbolístico: el caso de la actividad deportiva en español, y algunas propuestas de traducción al italiano" en L. Luque Toro (ed.) Léxico español actual. Venecia: Univ Venezia, Vol. II: 155-202.

Origgi, N.

2015. Lingua e terminologia nello sport: il caso del calcio. Varese: Univ. Insubria. Tesis Doctoral.

Ortega Arjonilla, E.

1995. Hermeneútica y traducción: esbozo de una teoría de la traducción a partir de las aportaciones de E. Goffman, E. Nida y P. Ricoeur. Granada: Univ. de Granada.

Pope, J. P.; Stewart, N. W.; Law, B.; Hall, C. R.; Gregg, M. J. y Robertson.

2015. "Knowledge Translation of Sport Psychology to Coaches: Coaches' Use of Online Resources" en International Journal of Sports Science \& Coaching, 10 (6): 1055-1070. 
Procházková, K.

2006. La traduction du livre L'Histoire du sport en Europe. Brno, Rep. Checa: Masarykova Univerzita, Filozofická Fakulta. Tesis Doctoral.

Richmond, S. A.; McKay, C. y A. Emery.

2013. "Knowledge translation in sport injury prevention research: an example in youth ice hockey in Canada" en British journal of sports medicine. 48: 941-942.

Ruiz, M. C. y A. P. Watt.

2012. "Translation and reliability of the preliminary Spanish version of the Sport Imagery Questionnaire" en Advances in Physical Education, 2 (2): 73-75.

Salvador, J. L.

2009. El deporte en Occidente: Grecia, Roma, Bizancio. Madrid: Cátedra.

Sartorio, F.; Vercelli, S.; Bravini, E.; Bargeri, S.; Moroso, M.; Plebani, G. y G. Ferriero.

2014. "Foot and Ankle Ability Measure: traduzione e validazione della versione italiana del modulo ADL (FAAM-I/ADL)" en La Medicina del Lavoro, 105 (5): 357-365.

Sonnenschein, W.

1980. "La literatura deportiva en Latinoamérica" Educación Física y Deporte, 2(2). Consulta: file:///C:/Users/fgarcos/Downloads/DialnetLaLiteraturaDeportivaEnLatinoamerica-5012089.pdf.

Stenling, C.

2014." Sport programme implementation as translation and organizational identity construction: the implementation of Drive-in sport in Swedish 
sports as an illustration" en International journal of sport policy and politics, 6 (1): 55-69.

Stiller, J., y D. Alfermann.

2007. "Die deutsche Übersetzung des Physical Self-Description Questionnaire (PSDQ)" en Zeitschrift für Sportpsychologie, 14(4): 149-161.

Walle, J. M. L.

2002. Adaptación al español del Inventario de Habilidades Psicológicas para el Deporte (PSIS R-5). Granada: Universidad de Granada. Tesis Doctoral.

Zeeck, A.; Leonhart, R., Mosebach, N.; Schlegel, S.; Linster, H. W. y A. Hartmann.

2013. "Psychopathologische Aspekte von Sport: Eine deutsche Adaptation der „Exercise Dependence Scale (EDS-R)" en Zeitschrift für Sportpsychologie, 20 (3): 94-106. 